\title{
Performance Evaluation of Hole AVOIDANCE TECHNIQUES IN GEOGRAPHIC FORWARDING FOR MOBILE AD HOC NETWORKS
}

\author{
Cynthia Jayapal ${ }^{1}$ and Sumathi Vembu ${ }^{2}$ \\ ${ }^{1}$ Department of Information Technology, KCT, Coimbatore, INDIA \\ cynthia_clementerediffmail.com \\ ${ }^{2}$ Department of ECE, GCT, Coimbatore, INDIA \\ sumi_gct20010yahoo.co.in
}

\begin{abstract}
Ad hoc networks prefer reactive routing over proactive routing due to the rapid changes in a nodes position. Geographic position based routing is a convenient method to route a packet from a source to a destination based on the local information alone. Geographic forwarding adopts a greedy routing technique that selects a forwarding node that is closer to the destination than itself. When a node cannot find any such neighbour, it is said to be in a hole and should recover to find an alternate path of delivery. The common recovery technique used in the literature is face routing. This paper compares the performance of face routing technique with two other recovery mechanisms proposed in the literature that uses limited flooding and directional flooding. It is found that sectoring is more efficient than topology and perimeter forwarding in terms of delivery rate, end to end delay, control overhead, average path length, and number of forwarding nodes.
\end{abstract}

\section{Keywords}

Geographic Forwarding, Mobile ad hoc networks, Greedy forwarding, Face Routing and Hole avoidance

\section{INTRODUCTION}

Mobile Ad Hoc Networks (MANETs) are infrastructure less network with mobile nodes that communicate with nodes within the radio range through the wireless link. The nodes that are not within the range communicate with the help of other nodes through multi hop communication. These devices can either move or remain stationary. They include a variety of devices such as cell phones, PDAs and laptops. Each node in an ad hoc network can send, receive and forward data packets. The topology of the network is dynamic, self configuring and self organizing. Routing in MANETs has moved from topology based to geographic position based. In proactive routing protocol, every node maintains one or more tables representing the entire topology of the network. To maintain the routing information, topology details must be exchanged between the nodes on a regular basis, increasing the state maintenance overhead but routes are always available on request. In reactive routing protocols the nodes do not initiate the route discovery process until a route to the destination is required. This leads to higher latency but has less overhead.

Geographic position-based routing relies on geographic position information. In geographic routing, no routing tables are to be maintained and there is no overhead to find or update routes, but it requires position information of mobile nodes. A source sends a message to the geographic location of the destination instead of using the network address. Geographic forwarding is a greedy routing algorithm. A forwarding node relays an incoming data packet to the neighbour which is nearest to the destination. A packet may also reach a node that does not 
know about any nodes closer than itself to the ultimate destination. The data packet cannot be forwarded any further. The packet is stuck in a local minimum where the forwarding set is empty. This is called a hole in geographic forwarding. The node where the packet is stuck is also called as a concave node [1].

To overcome this local optimum problem, there are different recovery mechanisms suggested in the literature. Most of the hole avoiding techniques available in the literature as given in Section 2, either uses face routing, or limited flooding or restricted directional flooding to avoid the hole while forwarding. We simulate and compare the performance of the following three techniques that uses hole avoidance mechanism in this paper. In face routing or perimeter forwarding [2], distributed planarization of the network graph is done and the recovery from greedy forwarding is by forwarding packets using the right-hand rule. In the right-hand rule a packet arriving on an edge at node $\mathrm{x}$, is forwarded on the next edge counter clockwise about $\mathrm{x}$ from the ingress edge. When a node whose location is closer to the destination than the source is found, the forwarding mode is switched back to greedy forwarding. In topology-based recovery [3], recovery from hole is achieved by expanded ring search method that uses limited flooding. A node sends request to its one-hop nodes. If a reply is received, choose that node as the next forwarding node. If no reply is received the searching range is increased till maximum number of hops. In Sectoring [4], each node in the network divides its neighbours into 16 sectors and informs its neighbouring nodes of its identification, position information and its own sectoring information. A node forwards packet according to its neighbouring nodes information (e.g. position, sectoring information) stored in the routing table. These techniques use geographic forwarding to forward packets towards the destination.

Chapter 2, reviews the related work in the area of hole avoidance in geographic routing. Chapter 3, describes greedy routing and the hole avoidance techniques chosen for performance comparison in this work. Chapter 4, gives the performance analysis of the hole avoidance mechanisms and chapter 5 , gives the conclusion.

\section{PRESENT WORK}

The various location based routing protocols available in the literature are discussed below. In location-based routing protocols, it is assumed that nodes know their own locations and the source node knows the location of the destination node. In order to obtain the location of the destination node, a location service is needed from which a node can query about the locations of other nodes. Most location-based routing protocols assume that a location service exists as an external resource. A node equipped with a Global Positioning System (GPS) receiver can obtain its own geographic coordinates [5]. When GPS support is unavailable, there are other localization techniques that mobile nodes can use [6, 7, 8, 9]. Li et al. [10], proposed GLS which is a scalable and robust location database that geographically addresses queries and registrations. Their system dynamically selects multiple database servers to store each node's location, for robustness against server failure.

Multicasting is an efficient means of data dissemination to a group. Most of the multicasting protocols use greedy geographic forwarding for relaying multicast packets to destinations. Position - based multicast (PBM) [11], uses the geographic position of the nodes to make forwarding decisions. This protocol recommends using greedy multicast forwarding to determine the next hop nodes based on the remaining distance of forwarding node to the destination. The forwarding decisions to choose a node close to the destination are made only on local knowledge and are hence considered to be scalable and robust against topological changes. The recovery from hole is by using face routing. 
Scalable position - based multicast (SPBM)[12], uses the information from global and local member tables provided by the group management scheme to make the forwarding decision that is based on the direction. It constructs a hierarchical quad tree to provide scalability and aggregation of membership information. To determine the most suitable next hop for a packet and a given destination, the source compares the geographic progress for each of the neighbours in respect to the destination and picks the neighbour with highest progress. The recovery from hole is by using face routing.

Efficient geographic multicast protocol (EGMP)[13], builds zone-based bi-directional tree and maintains the tree by introducing a concept called zone depth, which is the depth of the member zone and root of the tree. A packet is forwarded from upstream zone to downstream zone. The position information is used to guide the zone structure building, tree construction and packet forwarding. When an empty zone with no node is anticipated, the last core node that moves out of the zone sends the stored information to its upstream zone which connects itself to the downstream zones.

Geographic multicast routing (GMR)[14], is a location based algorithm that selects a route with least total distance to destination. It optimizes the cost over progress ratio, where the cost is equal to the number of neighbours selected for forwarding by reducing the remaining distances to destinations. GMR reduces packet transmissions by broadcasting the packet to all neighbours at each hop, but it has increased packet overhead as it includes the information about the selected neighbours in the packet header.

Hierarchical rendezvous point multicast (HRPM)[15], uses distributed geographic hashing to decompose a multicast group into subgroups of manageable size. Each zone has one Access Point (AP) and the entire region has one Rendezvous Point (RP) for each of the group. HRPM reduces the byte-overhead due to its hierarchical structure but is inefficient in terms of packet transmissions, as it uses unicasting the same data packet more than once within the tree. The membership management is done by AP and RP by handling the JOIN and LEAVE commands. The location update is threshold based. It constructs a source to AP overlay tree for delivery.

Hierarchical geographic multicast routing (HGMR) [16], seamlessly combines the scalability of HRPM and the forwarding efficiency of GMR. HGMR uses the same features of HRPM for the hierarchical decomposition and delivery from the source to the destination cell, but uses GMR's cost over progress tree for delivery within the cell. This takes the advantage from broadcasting a packet instead of unicasting it to each member. GMR, HRPM and HGMR uses face routing for recovery from hole.

Nadeem Ahmed et al. and Qing Fang et al. lists the techniques of avoiding holes in sensor networks [17,18]. GPSR [2] uses right hand rule using planar graph to avoid holes. Compass Routing [19], FACE-I, FACE-II[20] and GOAFR+ [21], uses face routing to avoid holes using planar graph. TENT rule [22], uses the boundary information of perimeter nodes to avoid the hole by checking for stuck angle. Near [23], predicts the dead ends which the greedy algorithm may reach and bypass voids in the network. If the angle between two adjacent node's neighbors exceeds 180 degrees, then the node is necessarily concave for routing in this direction. SOGR [3], uses expanded ring search technique to bypass the void areas.

\section{GREEDY ROUTING}

The wireless network is modelled as an undirected graph $G=(V, E)$ where $V$ is the set of vertices and $E$ is the set of edges. The model assumes that the network is two dimensional and wireless nodes are represented by vertices of the graph. Each node $v \in V$ has a transmission range $r$, which is equal for all nodes. Let $\operatorname{dist}(v 1, v 2)$ be the distance between two vertices $v 1$, 
$v 2 \in V$. An edge between two nodes $v 1, v 2 \in V$ exists $\Leftrightarrow \Rightarrow \operatorname{dist}(v 1, v 2) \leq r$ (i.e. $v 1$ and $v 2$ are able to communicate directly).

In geographic routing, a message can be routed to the destination without any knowledge of the network topology or a prior route discovery, with the help of the local information available dynamically. Every node maintains a neighbour table with the location information and node ID of all the neighbours in its transmission range that is updated with the help of periodical beacon messages.

\subsection{Geographical Forwarding}

Geographic forwarding [3], is a greedy routing algorithm commonly used by mobile nodes to transmit a data packet to the destination.

Algorithm

1. Find the position of the one hop neighbours of the source node

2. Choose a neighbour node which is closer to the destination than other nodes and the source node.

3. Send the packet to the chosen node.

Repeat steps 2 and 3 until the destination is reached.

The figure 1, shows the successful greedy forwarding where the source chooses $\mathrm{x}$ as the forwarding node as $\mathrm{x}$ is closer to destination than the source itself.

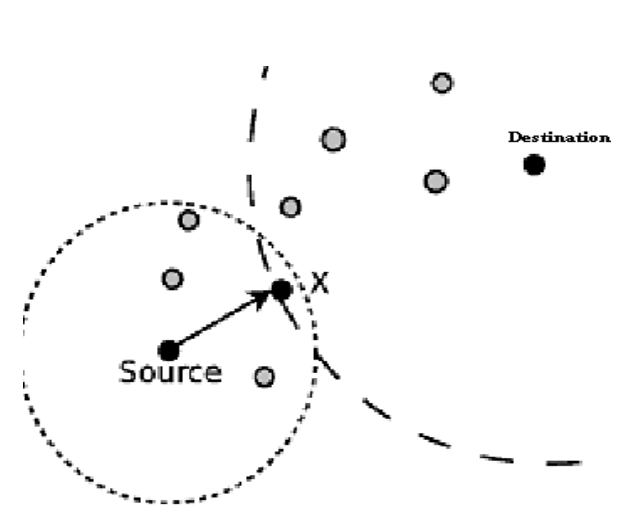

Figure 1. Greedy Forwarding

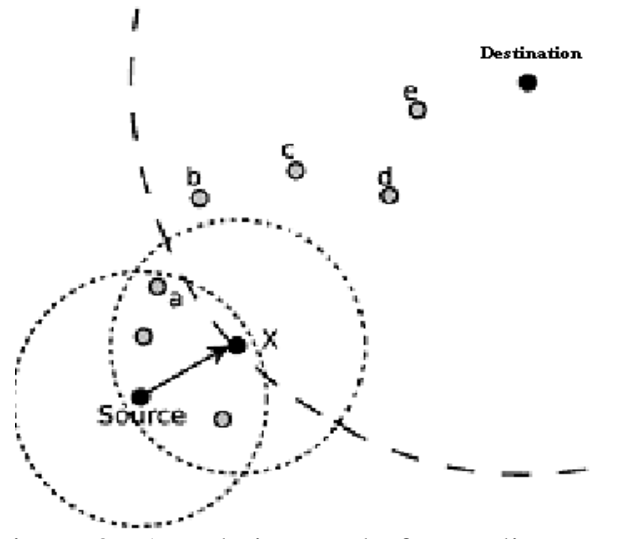

Figure 2. A Hole in greedy forwarding

Greedy forwarding does not always succeed in reaching a destination. A forwarding node chosen may not have any neighbour that is closer to the destination than itself and cannot forward the incoming data packet any further. This situation is called as a hole or empty zone problem. In Figure 2, the source node has chosen $\mathrm{x}$ as the forwarding node, but $\mathrm{x}$ could not forward the packet any further, because it does not have any node within its transmission range that is closer to the destination than itself and is said to be in a hole.

\subsection{Hole Avoiding Techniques}

The face routing, topology-based recovery and sectoring are the three hole avoidance techniques that has been chosen for performance comparison.

\subsubsection{Face Routing}

Face routing, proposed in [2], was the first geometric routing algorithm that guaranteed message delivery without flooding. Face routing is applied on a plane sub graph of the network graph. A plane graph divides the plane into faces. The line segment between the source node 
and the destination node intersects some faces. In face routing, the packet is forwarded along the boundaries of these faces. A typical face routing protocol works as follows [20]. A packet is forwarded along the boundary of the first face intersected by the line segment from the starting point to the destination. The first edge of the traversal of a face is the first edge in clockwise order around the starting point from the line segment to the destination. After the traversal of an edge $(\mathrm{x} ; \mathrm{y})$, the next edge of the face traversal is the first edge after $(\mathrm{y} ; \mathrm{z})$ in clockwise order around $\mathrm{y}$. In this way, the packet traverses the edges on the boundary of the face in the counter clockwise direction. The traversal in this way is called the right-hand rule. When the traversal reaches an edge that intersects the line segment from the starting point to the destination at a point closer to the destination than the starting point is, that point becomes the new starting point and the traversal switches to the next face. This procedure repeats until the destination is reached. The sequence of edges traversed by the right-hand rule is called as a perimeter. In Figure 3, x receives a packet from $\mathrm{y}$, and forwards it to its first neighbour counter clockwise about itself to $\mathrm{z}$.

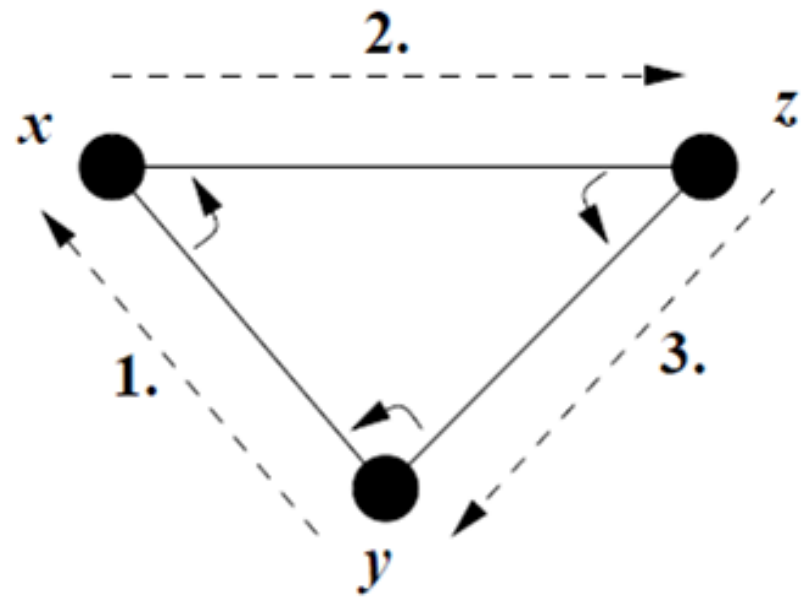

Figure 3 :The right-hand rule

\subsubsection{Topology Based Recovery}

In topology based recovery scheme proposed by Xiaojing Xiang et al.[3], a source node broadcasts request to its one hop neighbours with TTL value set to 1 ,to find a node which is closer to the destination than itself. If the destination is present in this request range, the destination acknowledges the source node and the data is sent from the source node to the destination node. Otherwise the neighbour node which is closer to the destination than the source node, acknowledges the source node and the TTL value is set to 0 . The neighbour node is then made as the current source node and the process continues till the destination is reached.

If the source node does not have any 1-hop neighbour node which is closer to the destination than itself, it broadcasts its request to its two hop neighbours by setting the TTL value as 2 . The 1- hop neighbours decrement the TTL value as 1 and then forwards the request. This search continues by incrementing the TTL value to the maximum number of hops, until a forwarding node or destination is found.

\subsubsection{Sectoring}

Geographic Routing with Neighbour Sectoring (GRNS) algorithm [4], like any other geographic routing algorithms uses geographic forwarding. It uses the sectoring technique to recover from a hole. 
Algorithm for sectoring

1. Each node in the network divides its neighbours into 16 sectors.

2. Each node informs its neighbour, the position and sectoring information which includes the number of neighbouring nodes in each sector.

3. When a node has a packet to send to a certain destination node, it will use the position information of its neighbouring nodes to calculate the distance from each neighbouring node to the destination node.

4. For each neighbouring node that is closer to the destination, the routing metric $\mathrm{F}$ is calculated as shown in equation (1).

$$
F=\frac{n^{*} D 1}{D 2}
$$

5. The neighbouring node that has the highest routing metric value for $F$ is chosen as the next hop node.

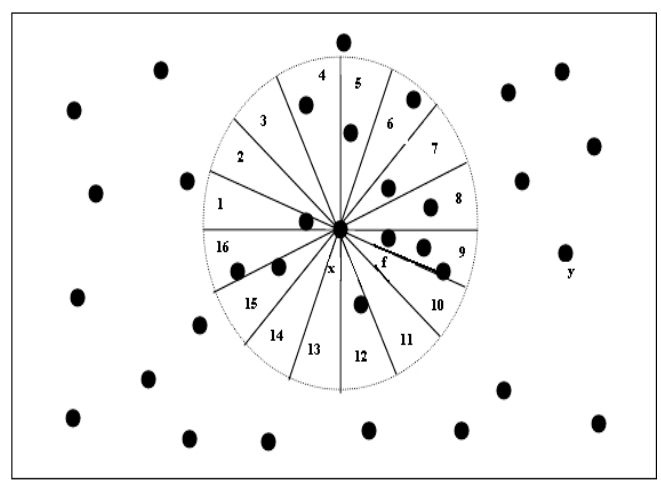

Figure 3. Sectoring

In Equation (1), $\mathrm{F}$ is the routing metric; $\mathrm{n}$ is the number of the neighbours to a neighbouring node. The $\mathrm{n}$ value can be calculated from the sectoring information of the neighbouring node in the current node's routing table. D1 is the distance from the current node to the destination. D2 is the distance from the neighbouring node to the destination node. A node will forward data packets to the neighbouring node with the largest routing metric F. This lowers the probability of forwarding a data packet to a dead-end node. A dead-end node has no neighbouring node closer to the destination node, and therefore its value of $n$ is 0 and consequently $F$ is 0 , so the data packet will not be forwarded to the dead-end node. If the destination node is in the range of a neighbouring node, the packet will be forwarded to that neighbouring node no matter what the value of $\mathrm{F}$ is.

\section{PERFORMANCE EVALUATION}

\subsection{Simulation Overview}

The geographic forwarding scheme was simulated using the wireless extensions developed at Carnegie Mellon [24]. The nodes are initially placed uniformly at random in a rectangular region of $1600 * 1600$. The radio range is $250 \mathrm{~m}$. We varied the node density from 20 to 50 . We disabled the random motion, so as to ensure that a hole is present. No retransmission is done. 
We averaged the simulation runs with different source and destinations. Each simulation lasts for 900 seconds of simulated time.

The following metrics were studied:

Packet delivery ratio: The ratio of the number of packets delivered to a destination to the number of packets sent by a source.

Average end to end delay: The average time interval for the data packets to reach a destination from the source.

Control message overhead: It is the total number of control messages needed for transmission of data packets.

Average path length: The average distance travelled by a packet that is transmitted from a source to a destination.

Average number of forwarding nodes: It is the average number of nodes that forward the packet from a source to a destination.

\subsection{Simulation Results}

The packet delivery ratio of sectoring is $41 \%$ greater than topology based forwarding and $47 \%$ greater than perimeter forwarding on an average. When network size increases the number of packets forwarded is more and hence the delivery ratio declines. The average end to end delay to recover from a hole is more or less same for all three techniques. The sectoring has $3 \%$ less delay than topology and $10 \%$ lesser than perimeter forwarding on an average. Average path length of sectoring is $8 \%$ less than topology and $17 \%$ less than perimeter forwarding on an average. In perimeter forwarding, the number of hops taken is more if the packet is forwarded in the wrong direction. The average path length increases when source and destinations are apart. The control overhead of sectoring is $11 \%$ lesser than topology and $16 \%$ lesser than perimeter forwarding on an average.

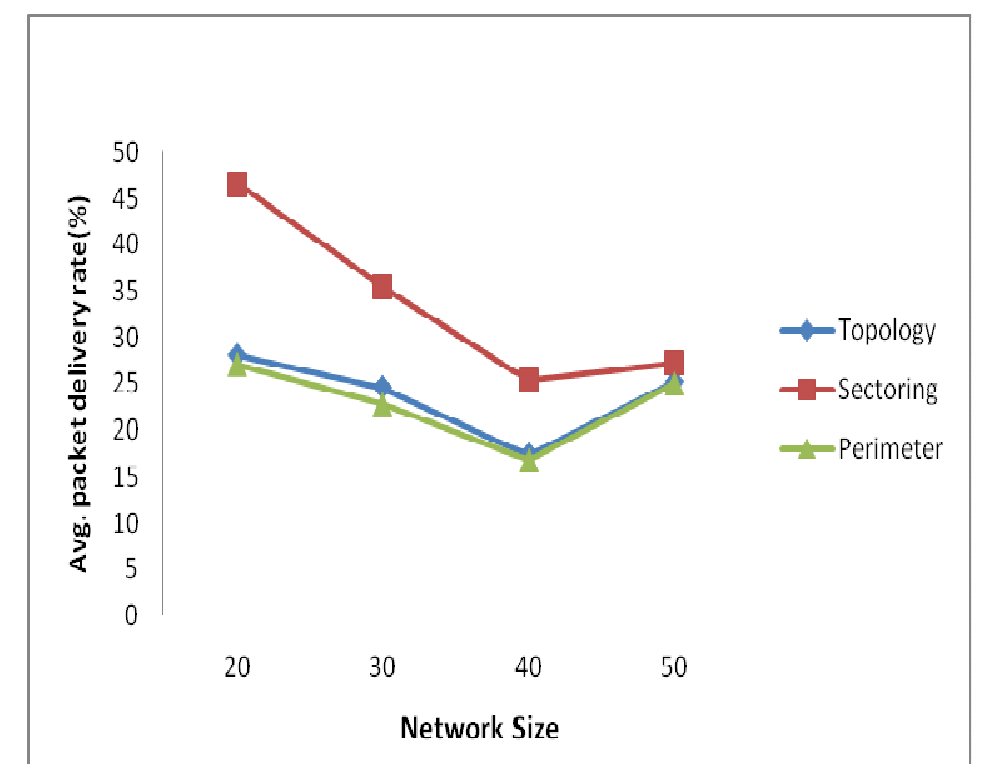

Figure 1. Effect of Network Size on Average Packet Delivery rate 
International Journal of Information Technology Convergence and Services (IJITCS) Vol.1, No.4, August 2011

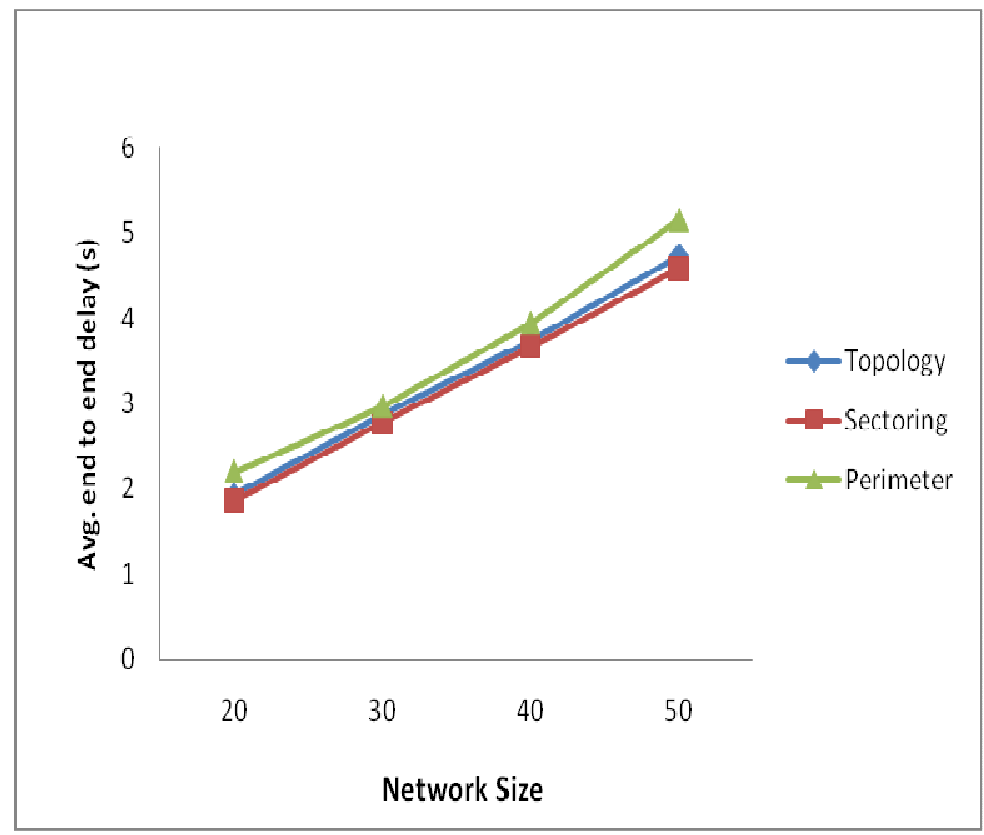

Figure 2. Effect of Network Size on Average end to end Delay

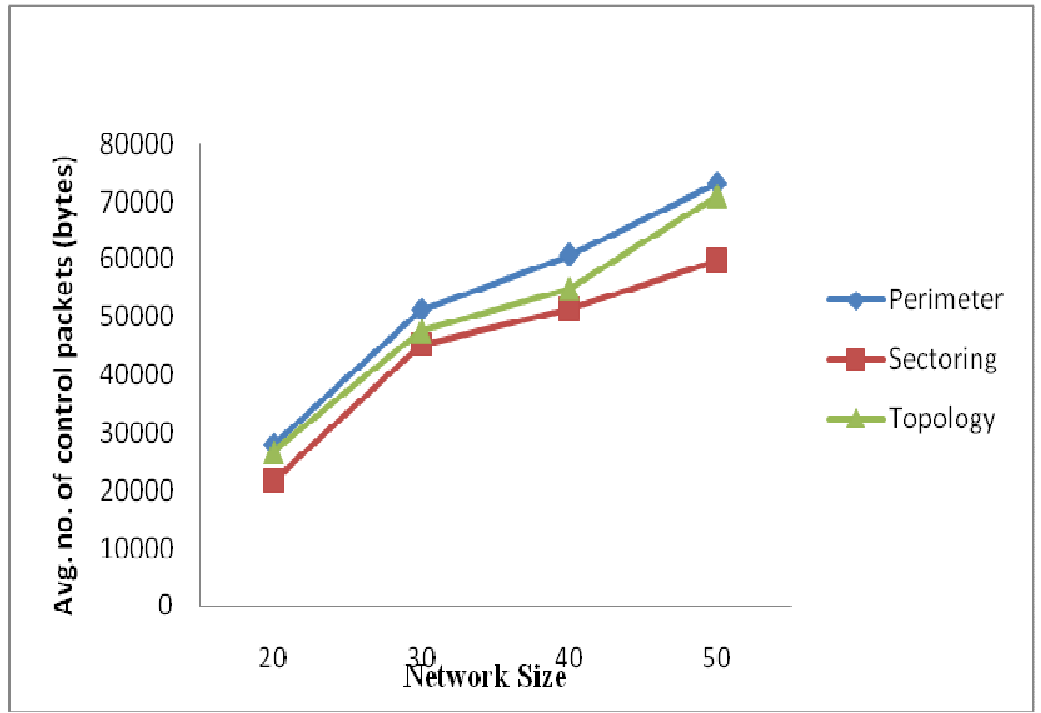

Figure 3. Effect of Network Size on Average number of control packets 
International Journal of Information Technology Convergence and Services (IJTCS) Vol.1, No.4, August 2011

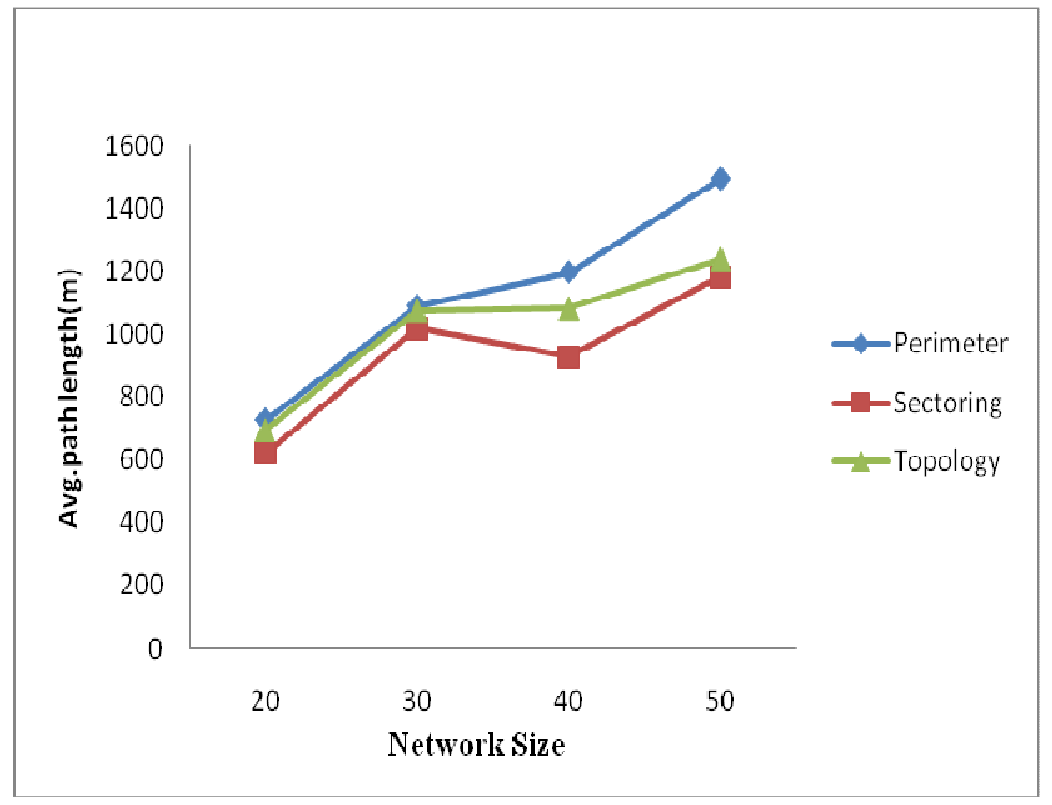

Figure 4. Effect of Network Size on Average Path Length

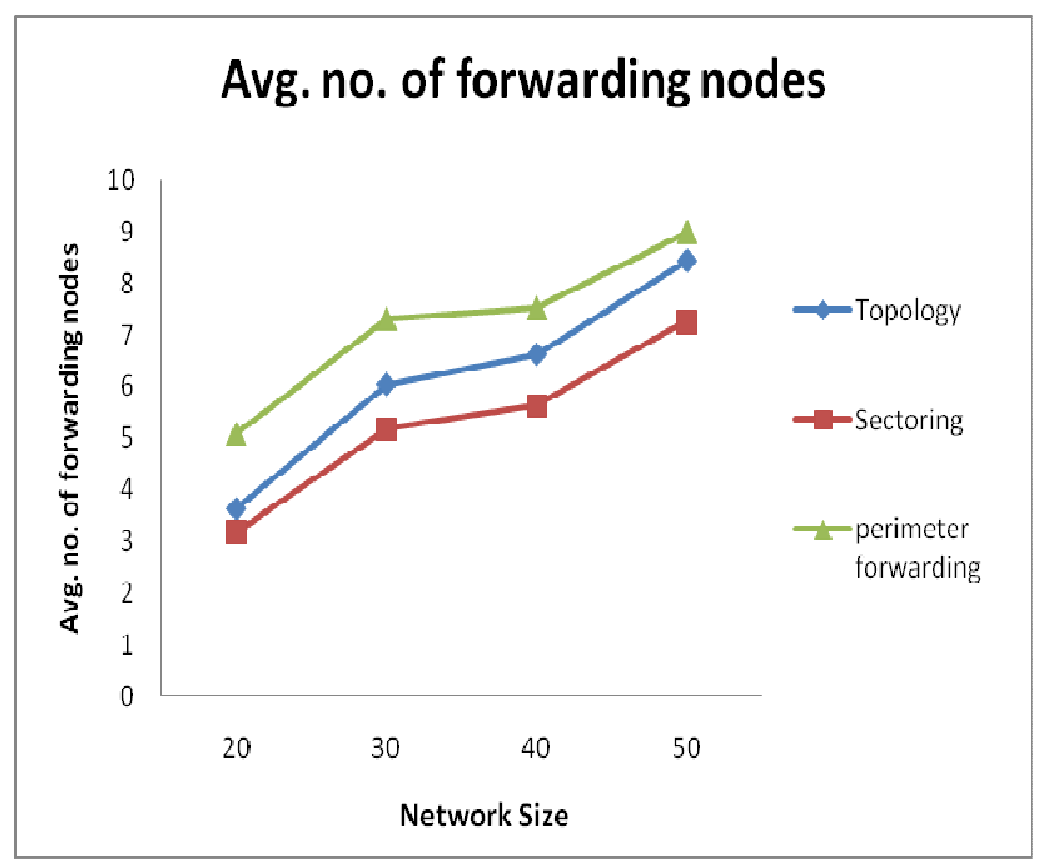

Figure 5. Effect of Network Size on Average number of forwarding nodes

\section{CONCLUSIONS}

A geographic routing technique uses greedy forwarding and a recovery technique, if the greedy approach fails in order to route a packet to the destination. The success of the recovery algorithm depends on how efficiently it is able to recover from the hole with the help of the 
local information available. In this work the greedy routing technique was simulated using ns 2 simulator. The hole that occurred during the greedy routing was recovered using face routing, topology-based recovery and sectoring. It was found that the performance of a directed routing technique like sectoring is better than the other two recovery schemes. Since the recovery mechanism involves more control overhead and latency in packet delivery, a routing algorithm must focus on choosing a route that avoids hole rather than recovering from a hole. As future work, we propose to develop a routing algorithm that chooses a forwarding node based on its location and remaining energy, so as to maximise the life time of the nodes in the forwarding set and avoid occurrence of a hole.

\section{REFERENCES}

[1] I. Stojmenovic, (2002)"Position based routing in ad hoc networks", IEEE Communications Magazine, Vol. 40, No.7,pp 128-134.

[2] Brad Karp \& H. T. Kung, (2000) "GPSR: Greedy perimeter stateless routing for wireless networks”, In Proceedings of ACM Conf. on Mobile Computing and Networking (MOBICOM), pp. 243-254.

[3] Xiaojing Xiang, Zehua Zhou \&, Xin Wang, (2007) "Self-Adaptive On Demand Geographic Routing Protocols for Mobile Ad Hoc Networks", In proceedings of IEEE International Conference on Computer Communication, pp 2296-2300.

[4] Jingren Jin,(2007) "Improving geographic routing with neighbour sectoring"(2007), Auburn Theses and Dissertations, Auburn University Alabama.

[5] Elliott D. Kaplan, (1996) "Understanding GPS: principles and applications", Artech House, Boston.

[6] Lingxuan $\mathrm{Hu}$ \& David Evans,(2004)“Localization for mobile sensor networks” , In Proceedings of the 10th annual international conference on Mobile computing and networking, pp 45-57.

[7] Nissanka B. Priyantha, Anit Chakraborty, \& Hari Balakrishnan, (2000) "The cricket locationsupport system", In Proceedings of the 6th annual International conference on Mobile computing and networking, pp 32-43.

[8] Andreas Savvides, Mani Srivastava, Lewis Girod, \& Deborah Estrin, (2004)"Localization in sensor networks". Wireless sensor networks, pp 327-349.

[9] Adam Smith, Hari Balakrishnan, Michel Goraczko, \& Nissanka Priyantha, (2004), "Tracking moving devices with the cricket location system", In Proceedings of the 2nd international conference on Mobile systems, applications, and services, pp 190-202.

[10] J. Li, J. Jannotti, D. S. J. D. Couto, D. R. Karger, \& R. Morris,(2000) “A scalable location service for geographic ad hoc routing”, In Proc. Of International conference on mobile computing and networking. pp.120-130.

[11] Martin Mauvae ,Holger Fubler, Jorg Widmer \& Thomas Lang, (2003), "Poster:Position Based Multicast Routing for Mobile Ad-Hoc Networks", In proc. Of The ACM International Symposium on Mobile Ad Hoc Networking and Computing, pp. 53-55.

[12] Matthias Transier, Holger FuBler, Jorg Widmer, Martin Mauve \&Wolfgang ffelsberg (2004), "Scalable Position -Based Multicast for Mobile Ad-hoc Networks" in First International Workshop on Broadband Wireless Multimedia: Algorithms, Architectures and Applications.

[13] Xiaojing Xiang, and Xin Wang,(2006), “An Efficient Geographic Multicast Protocol for Mobile Adhoc Networks", In Proc. of IEEE International Symposium on a World of Wireless, Mobile and Multimedia Networks , pp.73-82.

[14] Juan A.Sanchez , Pedro M. Ruiz \& Jennifer liu, (2007), " Bandwidth-Efficient Geographic Multicast Routing Protocol for Wireless Sensor Networks”, IEEE Sensors Journal, pp.627-636 . 
[15] Saumitra M.Das, Himabindu Pucha, \& Y.Charlie Hu,(2008). "Distributed Hashing for Scalable Multicast in Wireless Adhoc Networks", IEEE Transactions on Parallel and Distributed Systems. Vol. 19, No. 3, pp.347-362.

[16] Dimitrios Koutsonikolas, Saumitra M. Das, Y. Charlie Hu, \& Ivan Stojmenovic(2010), Hierarchical geographic multicast routing for wireless sensor networks. Wireless networks. Vol. 16, No.2, pp.449-466.

[17] Qing Fang, Jie Gao, \&Leonidas J. Guibas,(2004), ’Locating and bypassing routing holes in sensor networks", In Proceedings of IEEE International Conference on Computer Communications, vol. 4, pp 2458 - 2468.

[18] Nadeem Ahmed, Salil S. Kanhere \& Sanjay Jha,(2005),"'The holes problem in wireless sensor networks: a survey", ACM SIGMOBILE Mobile Computing and Communications Review, vol. 9, No. 2, pp 4-18.

[19] Evangelos Kranakis, Harvinder Singh, \& Jorge Urrutia(1999),”Compass routing on geometric networks", In Proceedings of the 11th Canadian Conference on Computational Geometry, pp51-54.

[20] P. Bose, P. Morin, I. Stojmenovic, \& J. Urrutia,(2001),’Routing with guaranteed delivery in ad hoc wireless networks"' Wireless Networks, 7,pp609-616.

[21] Fabian Kuhn, Roger Wattenhofer, Yan Zhong, \& Aaron Zollinger. (2003), “Geometric ad-hoc routing: Of theory and practice", In proceedings of 23rd ACM Symposium on Principles of Distributed Computing, pp.63-72.

[22] Q. Fang, J. Gao,\& L.J. Guibas,(2006),"Locating and Bypassing Holes in Sensor Networks”, Mobile Networks and Applications, vol. 11, No. 2, pp 187-200.

[23] Noa Arad \& Yuval Shavitt,(2009) "Minimizing Recovery State in Geographic Ad Hoc Routing”, IEEE transactions on mobile computing, Vol. 8, No.2,pp. 203-217.

[24] NS-2 Simulator, http://www.insi.edu/nsname/ns. Accessed 10 December 2008. 
International Journal of Information Technology Convergence and Services (IJTCS) Vol.1, No.4, August 2011

Authors

J. Cynthia received M.E. degree from Anna University chennai, in 2001. B.E degree in Computer Science and Engineering, in 1994. She is currently pursuing her research in the area of ad hoc networks in Anna University, Coimbatore. She is presently working as Associate Professor in the Deptt. of Information Technology at Kumaraguru College of Technology, Coimbatore, India. Her interests include, Data communication, routing and service provisioning in MANET. She has contributed over 20 technical papers in various journals and conference. She is a life member of ISTE.

V. SUMATHY, received Ph.D. in Engineering from Anna University in the year 2007. B.E and M.E degree in Electronics and Communication Engineering from Government College of Technology (GCT), Coimbatore, in the year 1988 and 2000 respectively. At present she is Associate Professor in the Department of ECE, GCT, Coimbatore. She has contributed more than 30 technical papers in Conferences and journals in

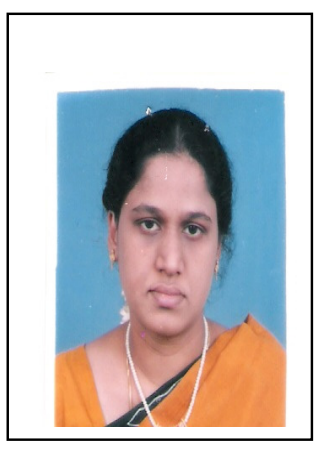
the area of Ad-Hoc Networks.

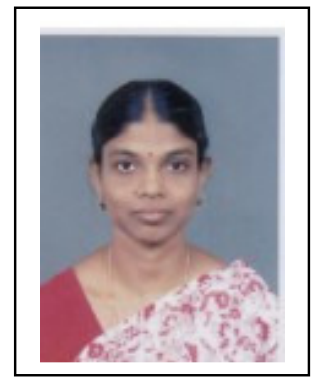

